\title{
Estimation of Creep and Cyclic Damage Accumulation in Micro-Turbine Disc
}

\author{
Alexander O. Chernyavsky ${ }^{a}$ \\ South-Ural State University, Russia
}

\begin{abstract}
The micro-turbine unit is intended to provide $100 \mathrm{~kW}$ of electric supply. The turbine is small size (turbine wheel diameter $150 \mathrm{~mm}$ ), high speed (65,000 rpm), and should have big enough durability (60,000 hours). Creep in stationary regime and cyclic inelastic deformation due thermal stresses at start up and stop restricts the turbine lifetime. Numerical analysis of temperatures, the creep strains and cyclic strain range shows that points with maximum creep damage and cyclic damage are different. This fact allows estimating the structure lifetime without exact knowledge of summation rule for the damages.
\end{abstract}

\section{Introduction}

Designed micro-turbine unit is intended for de-centralised electrical supply, output electric power is $100 \mathrm{~kW}$. The fuel is natural gas, chemical energy converts into mechanical (rotation of the electric generator rotor) by radial turbine. Estimated parameters of the turbine are [1]: nominal rotational speed $65000 \mathrm{rpm}$, life 60,000 hours, the gas temperature at the turbine inlet $1173 \mathrm{~K}$, outlet $-873 \mathrm{~K}$.

One of the most stressed parts of the unit is turbine disc, subjected to high temperatures, centrifugal forces, and variable thermal fields during the turbine start-stop. Strength and lifetime of the turbine disc in these conditions is restricted by a number of limit states:

- creep strain accumulation and quasi-static damages caused by the creep;

- accumulation of cyclic damages caused by variable thermal stresses (and inelastic strains) at "start-stop" cycles;

- possible residual displacement accumulation due to nonisochronic character of inelastic deformation in differrent parts of the structure ("incremental collapse" or "ratcheting");

- vibrations and high-cycle fatigue, material degradation in aggressive gas environment, etc.

The paper deals with the limit states connected with the inelastic strains (including creep) and their influence on the structure durability.

\section{Creep and long-term strength}

Circumferential speed of the disc edge at steady state is $510 \mathrm{~m} / \mathrm{s}$, which corresponds to high enough stresses: the elastic stresses for hypothetical disc of constant thickness exceed $850 \mathrm{MPa}$ (according to the formulas given in [2]).

\footnotetext{
${ }^{\mathrm{a}}$ Corresponding author: a.o.cher@mail.ru
}

Creep of the material leads to stress redistribution and decrease. If creep sufficiently developed to provide full stress redistribution, then the given angular speed corresponds to the stress level of $686 \mathrm{MPa}$ ("limit equilibrium" - see, for example, [3]). For such equipment russian strength codes demand a safety factor against burst speed not less than $n_{\omega}=1.3$ (a bit higher than 1.2 demanded by $[4,5])$. To ensure the required safety factor, a material of the constant thickness disc must have longterm strength not less than $1160 \mathrm{MPa}$.

Figure 1 shows the long-term strength of some currently used turbine alloys [6,7]. The abscissa of the graph is dimensionless parameter

$$
f=T(20+\lg (t))
$$

where $\mathrm{T}$ is Kelvin's temperature and $t$ - time in hours. For the designed unit with projected lifetime $t=60000$ hours of operation and the gas temperature at the turbine $T=873 \ldots 1173 \mathrm{~K}$ the $f$ parameter takes values 21000 to 29000 . Figure 1 demonstrates that the long-term strength of existing materials in these conditions significantly lower than necessary for the constant thickness disc, and ensuring of the durability demands appropriate geometry of the disc.

After a series of numerical tests, the disc with lightweight peripheral part (Figure 2) was proposed. To estimate creep and long-term strength, temperature field of the disc was calculated using finite-element analysis (ANSYS); temperature contours are shown on Figure 2. The calculations use the following inputs: heat exchange with the environment is convectional only; blades and the adjacent surface of the disc surrounded by combustion gases with temperature varying linearly along the radius from $900^{\circ} \mathrm{C}(1173 \mathrm{~K})$ at outer radius to $600^{\circ} \mathrm{C}(873 \mathrm{~K})$ at inner one, the heat transfer coefficient on this surface is 
$170 \mathrm{~W} / \mathrm{m}^{2} \mathrm{~K}$; back surface is surrounded by cooling air with a temperature of $250^{\circ} \mathrm{C}(523 \mathrm{~K})$ and heat transfer coefficient $500 \mathrm{~W} / \mathrm{m}^{2} \mathrm{~K}$.

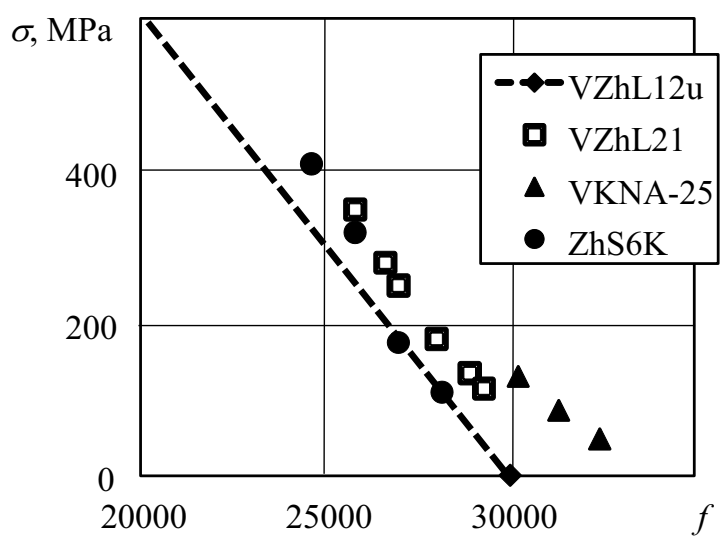

Figure 1. Long-term strength of 3 some nickel alloys depending on parameter $f$.

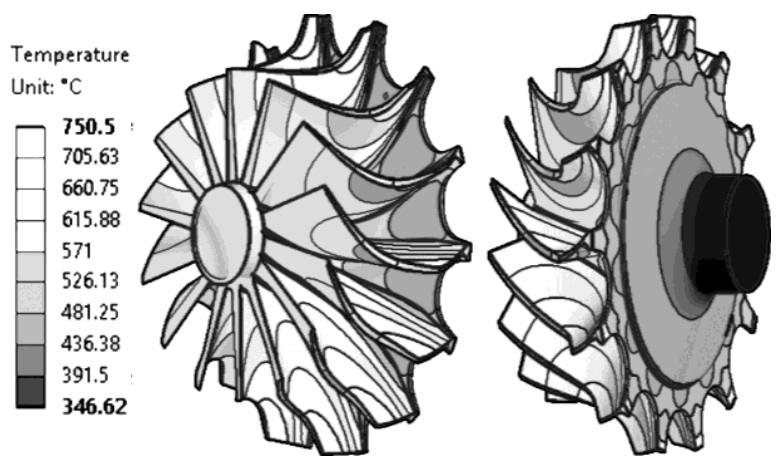

Figure 2. The disc outline and calculated temperatures

Existing creep data appears to be not suitable for reliable strain-stress calculation because of two reasons. First, usual handbook data (see, for example, [7]) deals with secondary stage of creep - not with the tertiary, necessary for failure analysis. Second, extrapolation of creep strain rates for a time, exceeding experimental more than 10 times, leads to significant errors - much greater than extrapolation of long- term strength. With this in mind, it was decided to replace a creep kinetic calculation by calculation using isochronous creep curves, representing the material as perfectly elastic-plastic with "yield strength" equal to long-term strength (for appropriate temperature and time). It is easy to see that such analysis corresponds to creep strain calculation, using a vertical line on the "strain vs time" graph as an approximation of tertiary creep.

Numerical analysis was done by ANSYS finiteelement software. Angular speed was increased step by step to find the burst speed, at which derivative of displacement with respect to time tends to infinity. Thermal strains - small enough - could not influence the results of such calculation, so only centrifugal forces were taken into account. Such approach coincides with static theorem of limit analysis theory (see, for example, [3]) and gives a lower (underestimating) value for the safety factor.

Figure 3 shows the distribution of the creep strain rate at a moment just before the solution become unconverged due to high strain and displacement (i.e. near the limit state). In accordance with the theory of limit equilibrium, this distribution is determined only to within an arbitrary constant factor, so Figure 3 illustrates the fracture mechanism, but not the exact values of accumulated strains. For VZhL21 alloy (Figure 1) estimated safety factor $n_{\omega}=1.24$ is lower than demanded value 1.3.

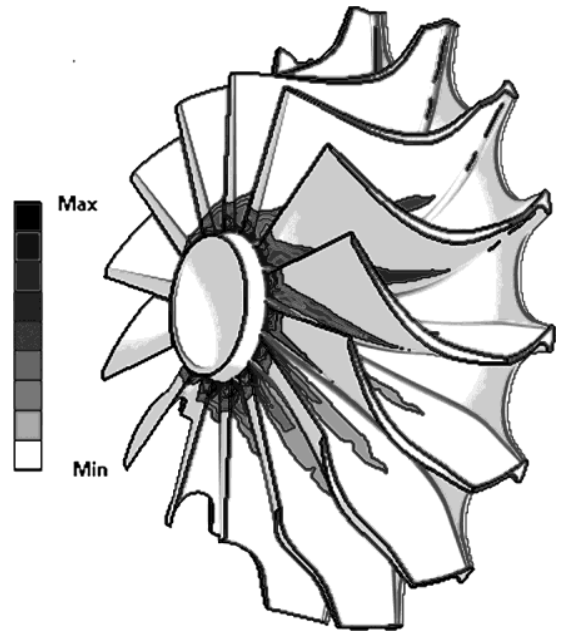

Figure 3. Creep strain rates at limit state

To achieve the required safety factor $n_{\omega}=1.3$ designers offer to change the geometry of the turbine wheel, deleting some material at the disc back surface (blades and front surface become unchanged) - Figure4. Decrease of the mass itself weakly affects the safety factor, because the removed mass was located near the axis of rotation and produces a relatively small centrifugal forces. But those changes in the disc geometry provide better cooling of the disc by ambient air and increase in long-term strength. Sensitivity analysis demonstrates that a uniform temperature reduction increases safety factor on $\Delta n_{\omega}=0.12$. Calculated temperatures for improved disc geometry, shown on Figure 4, demonstrates some temperature decrease comparing to the original structure on Figure 2 (darker color corresponds to lower temperature). Changes in temperature and increase in long-term strength (in accordance with the diagram of Figure 1) made it possible to achieve the required safety factor $n_{\omega}=1.3$.

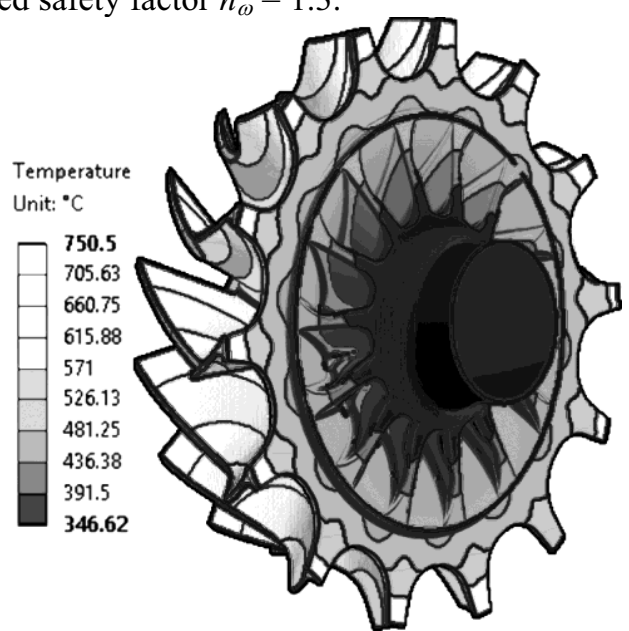

Figure 4. Temperatures calculated for improved disc geometry 
Achieving the desired safety factor become possible due increase of the material properties in the most loaded area and thus due a more uniform distribution of "stress to strength" ratio through the structure volume. This redistribution leads to changes in predicted fracture mechanism (Figure 5) and increase in masses of potentially separated fragments. Taking into account the operating conditions (people resides near the unit), it requires a safety assessment - analysis of opportunity to stop the fragments by surrounding parts of the unit or by a special safety structure.
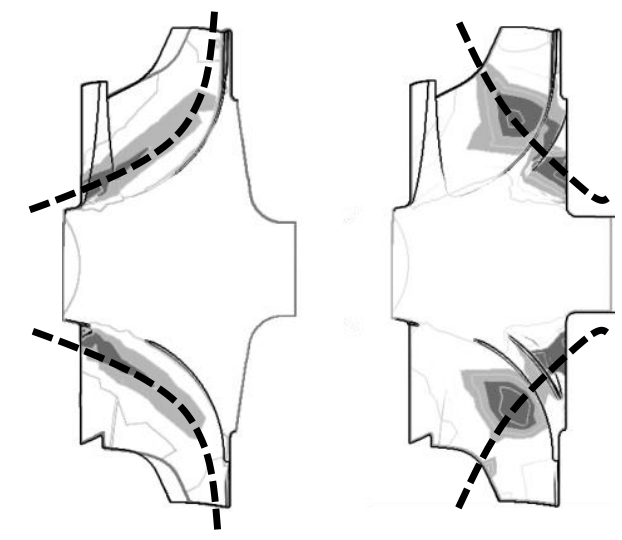

Figure 5. Predicted fracture modes for "original" and "improved" structures.

Darker color corresponds to higher strain rate. Dashed lines possible fracture surfaces.

\section{Cyclic loading}

The turbine start and stop produces transient stresses caused by varying mechanical and thermal loads. Mechanical loads include both centrifugal forces and torque generated by the electric starter. The latter is usually not taken into account because it creates small stresses. For the given structure a suspicion appears because gas bearings, designed for the unit, demands quick enough start to eliminate the bearing wear: the angular speed must reach $3000 \mathrm{rpm}$ (speed of stable gas layer formation) in not greater than 3 turns of the shaft. However transient finite-element solution, considering the electric starter torque characteristic, shows small enough stresses with maximum about $30 \mathrm{MPa}$. Such stresses do not restrict the structure life, because number of start-stop cycles cannot exceed $10^{5}$.

Thermal stresses in the start mode are much greater and can significantly influence the structure lifetime. To achieve upper estimation of the stresses the hypothetical case of "instant combustion chamber start" was adopted: the temperature of combustion gases changes stepped from 20 to $900^{\circ} \mathrm{C}$ (from 273 to $1173 \mathrm{~K}$ ). Transient temperature field was calculated using the same heat transfer coefficients as for the steady state before. Arrows on Figure 6 indicate places of the maximum thermal stresses. The stresses reach their maximum values after $10 \ldots 12$ seconds from the gas temperature change, later the stresses decreases due decrease in thermal gradients.

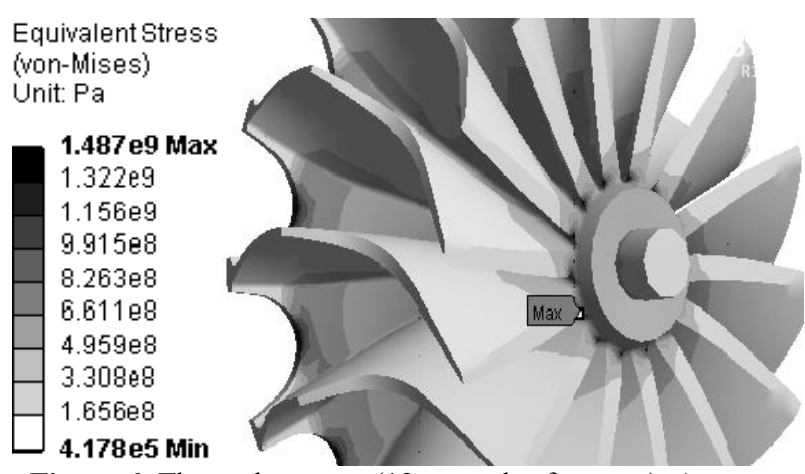

Figure 6. Thermal stresses (12 seconds after start). Arrows depict places of maximal stresses

Available literature does not contain the low-cycle fatigue characteristics of the VZhL21 alloy, used for the designed disc, so characteristics of the similar alloy ZhS6K [7] were used as a benchmark. Low-cycle lifetime was estimated using approach and safety factors regulated by the Strength code [8] (the safety factor is $n_{N}=10$ with respect to number of cycles and $n_{\sigma}=2$ with respect to conditionally elastic stresses). Estimated allowable number of cycles is 200 , which is certainly too little for the designed high-resource unit. This means that start-stop regimes demand a regulation, restricting thermal gradients and the stress levels. However this task is complicated by the conditions of combustion chamber stable work [9] and still is under consideration.

\section{Cyclic loading and creep interaction}

There are at least 3 aspects of the problem: mutual influence of the creep and cyclic loadin on mechanical behavior (hardening curve, cyclic hardening-softening, creep rate); summation rule for the cyclic and creep damages, and possible appearance of specific fracture mechanism: displacement accumulation due alternate plastic and creep strain increment (plastic - in transient start-stop regime, creep - in stationary one).

Some data on mechanical behavior of nickel alloys at a combination of creep and cycling are given in [10]. It was found that alloys with isotropic cyclic hardening demonstrate decrease of the hardening in case of creep, and for long enough cycles the cyclic hardening can be neglected: stress-strain diagram in arbitrary cycle is similar to the diagram in the first one (excluding some cycles prior the fracture). This fact simplifies calculation of stress-strain kinetics, eliminating the need for hardening accounting.

Summation rule for the creep and cyclic damages could be highly nonlinear (see, for example, [10] contrary to the linear summation rule according to the Code [8]). But exact rule, obviously, is not important for this particular task, because the strength of the structure restricted mainly by the long- term strength. It means that the creep damage is close to the limit value of 1 , and different summation rules give in this case close results (results in [10] demonstrate significant difference between linear summation rule and experiment mainly for the cases of comparable cyclic and static damages). 
Progressive displacement accumulation due alternate plastic and creep strain increment ("incremental collapse" or "ratcheting") can be estimated considering the "contraction" of the material yield surface at creep by the value, determined from the analysis of elastic-plastic deformation at start-stop. The Code [8] describes this procedure in detail, but for shell structures mainly. Paper [11] demonstrates that such type of deformation can be realized in a disc even without mechanical (centrifugal) load - at thermal actions of certain types. Preliminary analysis for the disc under consideration shows, that stresses, caused by thermal gradients at start-stop, and caused by centrifugal forces have different directions and signs (Figure 7), and thermal stresses can decrease the "yield surface" (i.e. the creep limit) and increase the accumulated strain. However exact analysis of this fact demand more precision creep description that used in this paper.

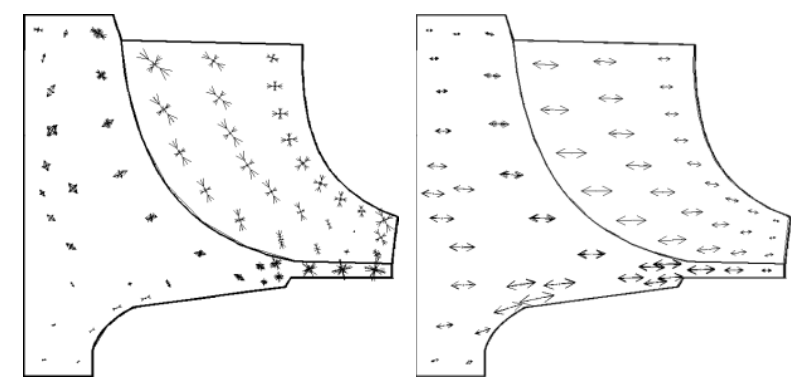

Figure7. Principal stresses, caused by thermal gradients and by centrifugal forces (one half of the disc cross-section and a blade).

\section{Conclusion}

1. The improved disc geometry provides necessary lifetime with nominal safety factor $n_{\omega}=1.3$. The main reason of difference in the safety factor between "source" and "improved" disc geometry is changes in cooling conditions: increased cooling surface in the second case provides some decrease in temperature, corresponding to increase in long-term strength. Decrease of the disc mass and changes in the volume of material subjected to high tensile stresses are less important.

2. Fracture mechanism for the disc can produce large enough fragments with high kinetic energy and so demands a safety analysis, proving the ability of the surrounding structure to stop the fragments and exclude people injury opportunity. Such analysis is planned as a next stage of the unit design.

3. Transient thermal stresses limit cyclic durability by a small enough value of 200 cycles in a case of "stepped" change in temperature of combustion gases from room temperature to the temperature characteristic for the nominal exploitation conditions. This means that thermal gradients on the start regime must be restricted and a procedure of "gradual" start must be developed. Such development, however, is not simple because of numerous additional restrictions connected with the work of gas bearings, stability of combustion process, critical frequencies of the rotor, etc.
4. Effects of possible interaction of creep and cyclic deformation were examined but found to be nonsignificant for the structure under consideration. This question should be possibly re-considered after the gradual start regime will be worked out.

The work was conducted with the financial support of the Ministry of Education and Science of the Russian Federation in the framework of the complex project "Establishing Production of New Generation Micro Turbine Units Range" according to the contract no. 02.G25.31.0078 d.d. 23.05.2013.

\section{References}

1. Degtyar B.G., Kartashev A.L., Martynov A.A. Research of operating regime of power micro gas turbine installation / Bulletin of the South Ural State University, Series "Mechanical Engineering Industry". vol. 13 (2013), no. 2, pp. 132-135

2. Boyarshinov, S.V.: Fundamentals of the Structural Mechanics of Machines. Russia, Moscow, "Engineering" publ., (1973) - 456 pp.

3. Cherniavsky O.F., Getsov L.B. Strength calculation for GTU members using shakedown theory // Proceedings of Scientific Production Association for Research and Design in Power Equipment named after I.I.Polzunov. - (2009) № 296. pp 253-260

4. EASA: CS-E 840 - Rotor Integrity - Certification Specifications for Engines, (2009).

5. FAA: FAR 33.27 - Turbine, compressor, fan, and turbosupercharger rotor overspeed .Federal Aviation Regulations, (2011)

6. High-Temperature Nickel Alloys of Low Density / Petrushin N.V., Ospennikova O.G., Visik Y.M., Rassokhina L.I., Timofeyeva O.B. // Liteinoye Proizvodstvo (Foundry. Technologies and Equipment) (2012) - № 6. - P. 5-11.

7. Mechanical properties of steels and alloys under unsteady loading. (handbook) / D.A.Gohfeld, L.B.Getsov, K.M.Kononov et al. - Ekaterinburg: Ural Branch of RAS, (1996) - 408 p.

8. Equipment and pipelines strength analysis norms for nuclear power plants PNAE G 7-002 86 - Russia, Moscow, Energoatomizdat publ., (1989) - 526 p.

9. Karpov A.I., Korepanov M.A., Salich V.L., Shaklein A.A. A gas dynamics and heat transfer simulation in the combustion chamber of the gas turbine power plant - Chemical physics and mezoskopiya -vol. 15 (2013). № 4. p. 530-536.

10. Makhutov N.A., Gadenin M.M., Evropin S.V., et al. Specific features of elastoplastic deformation and fracture of steels at complicated thermomechanical stress paths // Inorganic materials, Vol. 50 (2014), Issue 15 , p. 1495-1505

11. Chernyavskii O.F. Limiting states of turbomachine disks under conditions of high-intensity thermal changes // Journal of Machinery Manufacture and Reliability - Volume 43 (2014), Issue 6, p. 508-514 